\title{
Treatment of posttraumatic syringomyelia with extradural decompressive surgery
}

\author{
Langston T. Holly, M.D., J. Patrick Johnson, M.D., Jeffrey E. Masciopinto, M.D., \\ AND ULRICH BATZDORF, M.D. \\ Division of Neurosurgery, UCLA Medical Center, Los Angeles, California; and Rocky Mountain \\ Neurosurgical Alliance, Englewood, Colorado
}

\begin{abstract}
The authors review the management of five patients with posttraumatic syringomyelia (PTS) associated with an uncorrected spinal deformity. Patients with evidence of progressive neurological deterioration underwent ventral spinal decompressive surgery.

The mean patient age at the time of injury was 39 years, and the time between injury and the diagnosis of PTS ranged from 2 to 22 years. Mechanisms of injury consisted of fracture/subluxations in three patients and burst fractures in two. All patients experienced delayed neurological deterioration consistent with PTS. Magnetic resonance imaging revealed ventral deformities, and the spinal canal stenosis ranged from 20 to 50\% (mean 39\%). All patients underwent ventral epidural spinal decompressive surgery to correct the bone deformity and restore the spinal canal.

The mean follow-up period was 38 months. The decompressive intervention was initially successful in treating the neurological deterioration in all patients. Symptoms resolved completely in four patients, and the other experienced neurological improvement. Postoperative magnetic resonance imaging revealed a reduction in the size of syrinx cavity in the patients whose symptoms resolved and no change in the remaining patient. Two patients required a subsequent second-stage posterior intradural exploration and duraplasty for recurrence of symptoms and/or syrinx.

Posttraumatic spinal deformity may cause spinal canal stenosis and alter subarachnoid cerebrospinal fluid (CSF) flow in certain patients. Ventral epidural spinal decompressive surgery may result in neurological improvement and a reduction of the syrinx cavity, avoiding the need for placement of a shunt or other intradural procedures. However, some patients will also require reconstruction of the posterior subarachnoid space with duraplasty if the ventral decompressive procedure achieves only partial restoration of the subarachnoid CSF flow.
\end{abstract}

\section{KEY WORDS • syringomyelia • fracture • stenosis • subarachnoid space • decompressive surgery}

Posttraumatic syringomyelia is a well-recognized cause of delayed neurological deterioration in spinal cord-injured patients. It is currently believed that, following direct injury to the spinal cord, development and propagation of a syringomyelic cavity is related to a partial or complete obstruction of normal CSF flow in the subarachnoid space. . $^{7-11,18,22,23}$

Surgical management is indicated in patients with progressive neurological deterioration in cases with an associated syrinx. Previously, surgical treatment for PTS consisted of myelotomy and draining the syrinx via a shunt to the pleural or peritoneal cavities. ${ }^{2,18}$ However, because of high failure rates and improved understanding of the pathogenic mechanisms involved, reconstruction of the subarachnoid space has now become the preferred surgical modality in many institutions. ${ }^{2,10,13,19}$ The procedure essentially reestablishes CSF flow in the subarachnoid space by performing lysis of adhesions and duraplasty.

Abbreviations used in this paper: $\mathrm{CSF}=$ cerebrospinal fluid; $\mathrm{MR}=$ magnetic resonance; $\mathrm{PTS}=$ posttraumatic syringomyelia; $\mathrm{SCI}=$ spinal cord injury .
These views led us to believe that, in patients in whom trauma-related spinal deformity has not been corrected, persistent extradural osseous spinal cord compression can similarly cause obstruction of subarachnoid CSF flow. This study is used to evaluate outcomes in patients with a fixed spinal deformity and a symptomatic syrinx who underwent extradural decompressive surgery for the treatment of syringomyelia.

\section{CLINICAL MATERIAL AND METHODS}

\section{Patient Population}

Five patients with posttraumatic syringomyelia associated with an uncorrected spinal deformity were evaluated at the UCLA Medical Center between 1993 and 1999 (Table 1). There were four men and one woman. The mean age at the time of injury was 39 years (range 29-45). The mechanism of injury was a motor vehicle accident in four patients and a fall in one patient. A spinal fracture occurred in all cases: two were in the cervical region, two in the thoracic spine, and one at the thoracolumbar junction. 
TABLE 1

Summary of patient demographics, radiological findings, and history

\begin{tabular}{|c|c|c|c|c|c|c|c|}
\hline $\begin{array}{c}\text { Case } \\
\text { No. }\end{array}$ & $\begin{array}{c}\text { Age }(y r s), \\
\text { Sex }\end{array}$ & Initial Injury/Level & $\begin{array}{c}\text { Canal } \\
\text { Stenosis }\end{array}$ & $\begin{array}{c}\text { Syrinx } \\
\text { Location }\end{array}$ & $\begin{array}{c}\text { Previoius } \\
\text { Therapy }\end{array}$ & Fixed Defecit & PTS Symptoms \\
\hline 1 & $45, \mathrm{M}$ & C-7 burst & $50 \%$ & $\mathrm{C} 2-\mathrm{T} 1$ & halo vest, SPS & C-7 quadraplegia & UE pain \& weakness \\
\hline 2 & $29, \mathrm{M}$ & C-7 burst fracture & $50 \%$ & $\mathrm{C} 5-\mathrm{T} 3$ & brace, bed rest & C-7 quadraplegia & UE pain \& weakness \\
\hline 3 & $40, \mathrm{M}$ & T5-6 fracture/subluxation & $50 \%$ & T3-9 & PIF & spastic paraparesis, ambulatory & LE weakness \& spasticity \\
\hline 4 & $38, \mathrm{M}$ & T5-6 fracture/subluxation & $25 \%$ & $\mathrm{~T} 1-7$ & brace, bed rest & T-6 paraplegia & UE pain \\
\hline 5 & $44, \mathrm{~F}$ & L-1 fracture/subluxation & $20 \%$ & $\mathrm{C} 2-\mathrm{L} 1$ & $\mathrm{PF}$ & foot drop & UE/LE weakness \\
\hline
\end{tabular}

* $\mathrm{LE}=$ lower extremity; $\mathrm{PF}=$ posterior fusion; $\mathrm{PFI}=\mathrm{PF}$ in which instrumentation was placed; SPS = syringopleural shunt; UE = upper extremity .

All of the spinal deformities were ventral and resulted in focal bone compression of the spinal canal at the level of injury. The mean time interval between the SCI and initial diagnosis of posttraumatic syringomyelia was 11 years (range 2-22 years). Three patients suffered injury-related complete neurological deficits and two, incomplete deficits.

\section{Preoperative Deficits}

Patients in Cases 1 and 2 had a C-7 functional level and presented with worsening upper-extremity motor function and burning pain. The patient in Case 3 presented with increasing lower-extremity spasticity and progressive deterioration in left lower-extremity strength. The patient in Case 4 sustained a complete SCI at T- 6 and presented with new onset of burning thoracic region and upper-extremity pain. The patient in Case 5 had a left foot drop after initial injury but developed ascending weakness that affected her distal lower extremities and right upper extremity.

\section{Surgical Treatment}

All five patients underwent surgical intervention to treat the onset of new neurological symptoms resulting from posttraumatic syringomyelia. In all cases, we endeavored to relieve the subarachnoid block by performing a ventral epidural spinal decompressive procedure at the site of the spinal deformity (Fig. 1). The patients were informed that management of their PTS may require a subsequent second-stage procedure in which the posterior subarachnoid space is reconstructed at a later date.

\section{RESULTS}

The results of surgery are summarized in Table 2 . The mean follow-up period was 38 months and ranged from 6 to 75 months. The patients underwent follow-up examination at 1,3 , and 12 months postoperatively. Preoperative and postoperative MR imaging was performed in all patients. Ventral spinal decompressive surgery initially yielded successful results in all five patients $(100 \%)$. In four patients complete neurological improvement of their previous post-SCI baseline status was demonstrated, and in the other patient (Case 5) improvement was shown but she did not return to her previous baseline status. The patient in Case 1 experienced a significant increase in intrinsic hand muscle strength, and her upper-extremity pain resolved. The patient in Case 2 similarly noticed a disappearance of upper-extremity pain, as well as a return of intrinsic hand muscle function. The patient in Case $3 \mathrm{ex}-$ perienced an increase in lower-extremity strength and a decrease in spasticity (Fig. 2). A recovery of bowel and bladder sensation and disappearance of pain were demonstrated in the patient in Case 4. These clinical outcomes correlated well with the postoperative MR imaging findings, which demonstrated a decrease in syrinx size in all patients except for that in Case 5. The kyphotic deformities and canal stenoses were significantly improved as well. No case of neurological worsening occurred, and there were no surgery-related complications.

The patient in Case 4 improved clinically for 1 year after undergoing decompressive surgery until he noticed a recurrence of upper-extremity weakness and pain. An MR image revealed reexpansion of the syrinx. Computerized tomography myelography demonstrated a web in the posterior subarachnoid space at the level of injury, which had not been evident on previous studies. Consequently he therefore underwent a T4-7 laminectomy in which intradural lysis of adhesions was performed. Arachnoidal adhesions were divided and partly excised around the

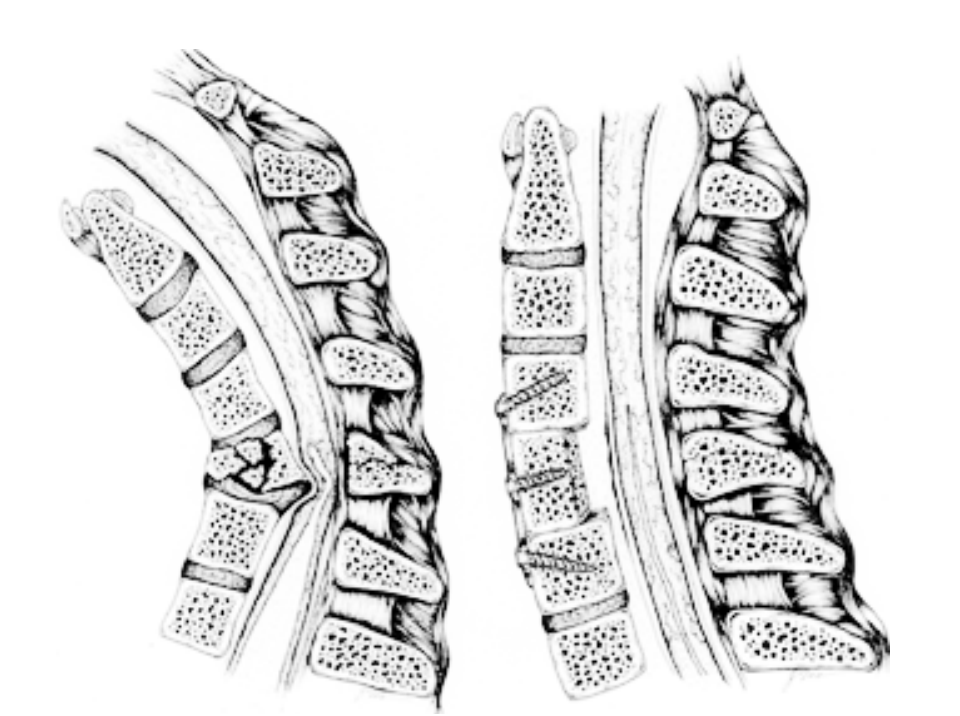

Fig. 1. Schematic drawing. A hypothetical C-5 burst fracture and development of a spinal cord syrinx is shown. Left: The displaced bone fragments compress the spinal cord and cause a functional obstruction of the subarachnoid space. A posttraumatic syrinx is shown below the level of injury. Right: Correction of the fracture and syrinx by a C-5 vertebrectomy and C4-6 fusion with bone graft and plating. The illustrated kyphosis and stenosis is shown to be improved, and the syrinx is shown as markedly reduced in size. Syrinx cavities may extend in either or both directions from the site of injury. 
TABLE 2

Summary of surgery-related treatment results

\begin{tabular}{|c|c|c|c|c|c|}
\hline $\begin{array}{l}\text { Case } \\
\text { No. }\end{array}$ & $\begin{array}{c}\text { IFI } \\
(\mathrm{yrs})\end{array}$ & Treatment & PTS Symptoms & Syrinx Size & $\begin{array}{l}\text { Follow Up } \\
\quad(\operatorname{mos})\end{array}$ \\
\hline 1 & 17 & C-7 vertebrectomy & resolved & decreased & 48 \\
\hline 2 & 5 & C-7 vertebrectomy & resolved & decreased & 75 \\
\hline 3 & 22 & T5-6 vertebrectomy & resolved & decreased & 6 \\
\hline 4 & 2 & $\begin{array}{l}\text { 1st op: T5-6 vertebrectomy; } \\
\text { 2nd op: posterior duraplasty } 1 \text { yr later }\end{array}$ & $\begin{array}{l}\text { resolved, but recurred } 1 \text { yr later; } \\
\text { resolved }\end{array}$ & $\begin{array}{l}\text { decreased, but recurred } 1 \text { yr later; } \\
\text { decreased }\end{array}$ & 14 \\
\hline 5 & 13 & $\begin{array}{l}\text { 1st op: L1 vertebrectomy; } \\
\text { 2nd op: posterior duraplasty } 4 \text { mos later }\end{array}$ & $\begin{array}{l}\text { improved; } \\
\text { improved }\end{array}$ & $\begin{array}{l}\text { no change; } \\
\text { decreased }\end{array}$ & 49 \\
\hline
\end{tabular}

*IFI = interval from injury.

right dorsal and lateral aspect of the spinal cord until it was freed, and the syrinx was decompressed using a 25gauge needle. An 8-cm autologous fascia lata graft was placed to expand the subarachnoid space. The patients symptoms resolved. In the patient in Case 5 partial neurological improvement was shown with some increase in upper-extremity strength, yet her lower-extremity strength did not improve significantly. At 4 months postoperatively, MR imaging revealed no appreciable change in syrinx size. She subsequently underwent a T1-L1 laminectomy in which intradural lysis of the adhesions and duraplasty were performed. The arachnoidal adhesions were sharply divided around the dorsal and lateral circumference until the spinal cord was freed of all visible adhesions. A $3-\mathrm{cm}$ autologous fascia lata graft was used for the duraplasty. Her follow-up MR images demonstrated a reduction in the size of her syrinx, and her lower-extremity strength improved.

\section{ILLUSTRATIVE CASE}

\section{Case 1}

History. This 45-year-old man suffered a motor vehicle-related C-7 burst fracture and quadraplegia 17 years

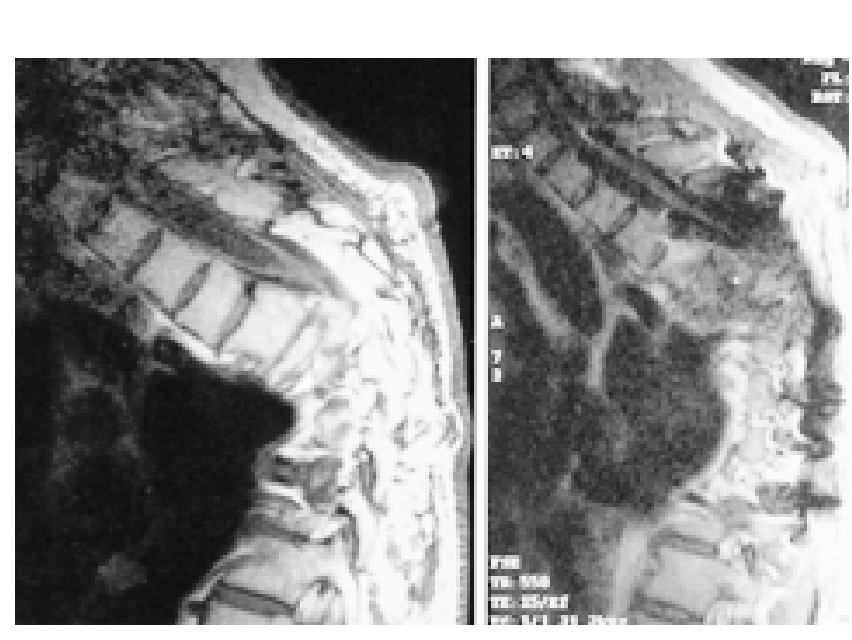

Fig. 2. Case 3. Left: $\mathrm{T}_{1}$-weighted MR image obtained in a patient in whom kyphosis developed after he suffered a T5-6 fracture/subluxation. A large posttraumatic syrinx is evident cephalad to the fracture. Right: An MR obtained following T5-6 vertebrectomy, revealing nearly complete disappearance of the syrinx cavity 4 months postoperatively. previously. He underwent cervical traction and a halo vest therapy, and the fracture healed. His neurological status remained stable for the initial 15 years postinjury, with a C-7 motor and sensory level that included some hand grip strength that enabled him to operate a wheelchair.

He then experienced progressive hand weakness, and lost the ability to operate his wheelchair. An MR image revealed a syrinx cavity, and he underwent placement of a syringopleural shunt at another institution. He experienced transient clinical improvement of his symptoms and was reported to have slight reduction of the syrinx cavity. He soon developed upper-extremity pain and suffered further deterioration in upper-extremity function. Repeated MR imaging demonstrated a large multiseptated syrinx cavity that extended from the inferior medulla to T-1, with a large retropulsed bone mass that narrowed the spinal canal by $50 \%$ at C-7 (Fig. 3 left). He was then referred to our institution for further management.

Surgery and Outcome.A cardiac-gated CSF flow study revealed an absence of flow around the spinal cord from C-3 to C-7. The clinical diagnosis was shunt failure and recurrent PTS. The surgical options considered were: 1) shunt revision, 2) posterior intradural exploration and duraplasty, or 3) correction of spinal deformity. Because of the focal and severe bone spinal canal lesion, he underwent a C-7 vertebrectomy to achieve an epidural decompression of the ventral spinal canal. Reconstruction of the spinal column was accomplished by performing a C6-T1 interbody fusion in which autologous iliac crest bone graft and instrumentation were used. Neurologically he experienced gradual improvement, with resolution of his upperextremity pain and weakness over several months following surgery. Postoperative MR images obtained 3 months after surgery demonstrated a marked decrease in the size of the syrinx and restoration of the spinal canal (Fig. 3 right). A repeated cardiac-gated CSF flow study revealed significantly improved flow in the subarachnoid space at the level of previous obstruction. The patient continues to do well 4 years postoperatively.

\section{DISCUSSION}

\section{Incidence and Features of PTS}

The true incidence of PTS remains unclear. Traditionally the medical literature indicates that the incidence is between $1 \%$ and $3 \%$ 3,5,6,7 of all patients with SCI. More contemporary estimates show that the incidence is closer 
to $10 \%,{ }^{6}$ and recent autopsy and MR studies suggest the incidence approaches $20 \% .{ }^{20} \mathrm{~A}$ major factor affecting this increased incidence is that more patients are surviving life-threatening accidents and have a longer life expectancy due to improved emergency medical services, intensive care, and rehabilitation care after sustaining severe SCI. Because symptoms of PTS are often delayed many years postinjury, more patients are now living long enough eventually to develop PTS.

Patients and physicians are now more alert to the classical signs and symptoms of PTS-pain, asymmetrical motor loss, spasticity, ascending sensory level, and hyperhydrosis. ${ }^{4}$ Should any of the aforementioned signs or symptoms occur, thorough medical investigation is required, as a diagnosis of PTS must be considered.

\section{Pathogenesis of PTS}

Many theories have been proposed to explain the formation and enlargement of posttraumatic spinal cord cysts, but most professionals generally accept that SCI is the initiating event. Scarring and adhesions occur within the subarachnoid space as the acute inflammation abates, ${ }^{19}$ initially causing at least a partial subarachnoid obstruction, particularly when combined with spinal cord edema and a narrowed spinal canal due to osseous impingement. It is postulated that this results in an increased intraspinal pressure due to CSF flow resistance at the site of obstruction in the subarachnoid space. Consequently, in the subarachnoid space, CSF, which encounters resistance to flow via the normal subarachnoid pathway may be driven transmurally into the spinal cord. ${ }^{21}$

\section{Evidence of Spinal Deformity and Spinal Cord Compression Causing Syringomyelia}

Extradural spinal cord compression is known to cause

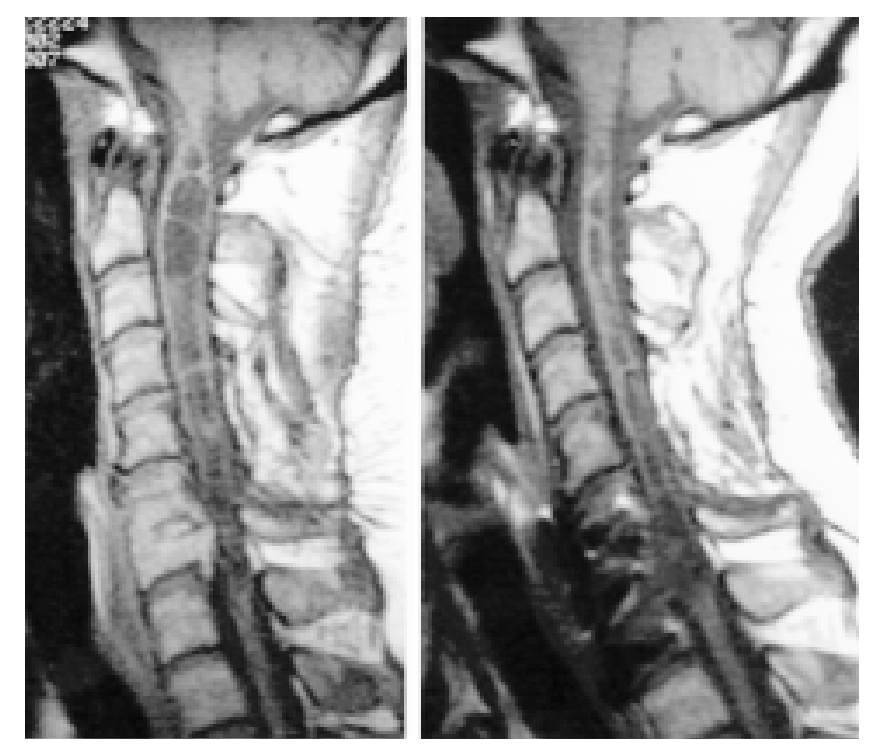

Fig. 3. Case 1. Left: $\mathrm{A} \mathrm{T}_{1}$-weighted MR image obtained in a patient with a C-7 burst fracture and canal stenosis with a large multiseptated syrinx. Right: An MR image obtained following C-7 vertebrectomy, spinal decompression, and stabilization with significant decrease in syrinx size 3 months after surgery. syringomyelia in several other well-known clinical entities likely due to the obstruction of subarachnoid CSF flow. In a series of 46 children, Menezes ${ }^{14}$ has reported that syringomyelia is caused by basilar invagination with extradural bone compression. Ventral extradural odontoid resection resulted in collapse of the syrinx cavities. Hormigo, et al., ${ }^{8}$ have described a patient who harbored an extramedullary lymphoma at the C1-2 level that caused syringomyelia. The patient underwent radiotherapy and steroid therapy that resulted in the disappearance of both the tumor and the syrinx cavity. Extradural lesions in the subaxial cervical spine (that is, focal disc herniation and narrowing of the subarachnoid space) causing formation of a syrinx have resolved following discectomy. ${ }^{15}$ These degenerative and congenital cases are particularly supportive of this theory because trauma is not a complicating factor. Therefore, the occurrence of an extradural lesion-associated syringomyelia and resolution of the syrinx after removal of this extradural mass suggests a direct causal relationship. The pathogenic mechanisms of these cases likely involve direct extradural compression that causes sufficient obstruction of the subarachnoid space to result in a syrinx cavity.

Despite the knowledge of the relationship between extradural spinal cord compression and syringomyelia, relatively little attention has been directed to the potential importance of relieving the spinal cord compression by correcting the spinal deformity in selected cases of PTS. In an extensive review of the management of PTS conducted by Edgar and Quail, ${ }^{4}$ the authors discussed surgical options for PTS but did not include correction of spinal deformity. Similarly, Schurch and associates ${ }^{17}$ reviewed 449 cases of SCI, and only two cases were briefly described in which surgical correction of a spinal deformity were associated with collapse of a syrinx cavity.

\section{Relationship Between PTS and Stenosis of the Spinal Canal}

It is extremely difficult to predict which spinal cordinjured patients will develop PTS based on age, sex, level of injury, or initial neurological deficit. ${ }^{19}$ Similarly, there is no proven relationship between the severity of the SCI and the development of syringomyelia. ${ }^{12}$ However, spinal fractures causing deformity have been implicated by Williams ${ }^{22}$ as being one of many factors in the pathogenesis of PTS. ${ }^{19}$

In a review of 75 spinal cord-injured patients, PerrouinVerbe, et al., ${ }^{16}$ evaluated the relationship between stenosis of the spinal canal caused by insufficiently reduced spinal fractures and the occurrence of PTS. In a total of 21 of their patients they found evidence of syringomyelia. They determined that the percentage of spinal canal stenosis was significantly higher in patients with a syrinx than in those without. Moreover, the incidence of syringomyelia is significantly higher in patients in whom the stenosis is greater than $30 \%$ in the sagittal plane and $20 \%$ in the axial plane, as demonstrated on MR imaging. The results of this study were validated by Abel, et al., ${ }^{1}$ who evaluated a series of 207 spinal cord-injured patients and determined that patients with greater than $25 \%$ spinal canal stenosis were twice as likely to develop PTS. These reports correlate well with the findings in our series that spinal de- 
formity has a significant relationship to the pathogenesis of PTS.

\section{Syrinx Reduction and Clinical Outcomes}

In our experience in the operative treatment of approximately 70 patients with PTS, we believe that because of the substantial heterogeneity in this population, individual evaluation and management are required. Extradural decompressive surgery was initially successful in all five of our current patients. Neurological improvement was attained in all patients in whom we performed epidural decompression, and postoperative MR imaging revealed a significant decrease in size of the syrinx cavities in the four patients whose symptoms resolved. Similarly, other authors have found a direct correlation between the reduction in the size of syrinx visualized on postoperative MR imaging and the patient's clinical outcome. ${ }^{5}$ However, none of of the syrinx cavities disappeared completely, which indeed may not be achievable in many patients treated for syringomyelia. In some patients this may be due to circumferential subarachnoid scarring, which prevents complete collapse of the syrinx. Another contributing factor may be tissue compliance in which the stiffness of the spinal cord parenchyma does not allow full collapse of the syrinx.

Two patients in our series, in whom we documented initial clinical and radiological improvement following ventral decompression, experienced recurrent neurological deterioration in conjunction with MR imaging-documented syrinx reaccumulation. Both patients underwent procedures in which the posterior subarachnoid space was reconstructed. The patient in Case 4 had made good recovery initially, experiencing decreased pain and improved lower-extremity motor and bladder function. The syrinx cavity was significantly reduced, but unfortunately he deteriorated clinically with recurrence of the syrinx 1 year later. Myelography revealed that the posterior subarachnoid space was obstructed at the level of injury, a finding not apparent on his preoperative myelogram. The arachnoidal adhesions were likely present at the time of his ventral procedure, but the correction of the bony deformity was initially sufficient to allow collapse of the syrinx. However, the posterior arachnoidal webbing presumably was sufficient to cause recurrent obstruction, which led to reformation of the syrinx. The patient in Case 5 experienced a partial neurological improvement after her ventral epidural decompressive procedure, but MR imaging did not demonstrate any appreciable change in the size of the syrinx. This is an example of when corrective surgery of a spinal deformity fails to achieve adequate subarachnoid decompression, likely because subarachnoid scarring is too extensive to respond to this management. The patient underwent posterior subarachnoid space reconstruction using microdissection and duraplasty 4 months later, and further resolution of symptoms and syrinx reduction were obtained.

We did not include in our series a patient with PTS and an uncorrected T-10 spinal deformity who presented with a spinal cord syrinx cavity both above and below the site of compression. Reconstruction of the posterior subarachnoid space was performed prior to our treating patients with ventral thoracic epidural procedures. He initially im-

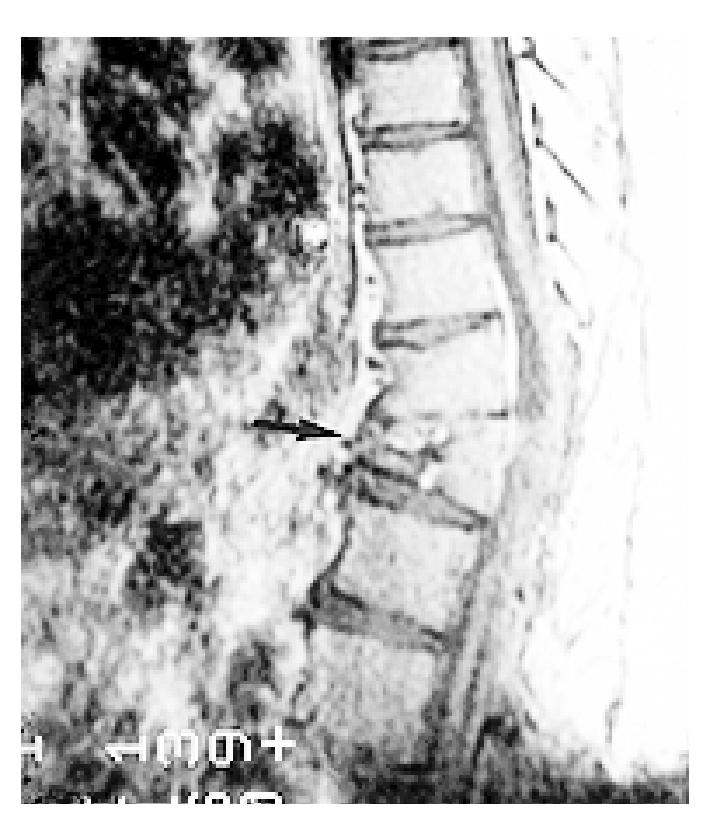

Fig. 4. Magnetic resonance image demonstrating an unreduced T-10 compression fracture (arrow) in a 40-year-old man; he underwent posterior subarachnoid space reconstruction for treatment of a T5-12 syrinx without ventral decompression. The syrinx reexpanded below the fracture site, and he deteriorated clinically.

proved neurologically, and the rostral and caudal syrinx was reduced in size. However, 1 year later he worsened clinically, and MR imaging demonstrated refilling of the syrinx caudal to the spinal cord deformity (Fig. 4). Despite decompressing the canal posteriorly, the spinal cord appeared as though it remained draped over the remaining ventral deformity. Sgouros and Williams ${ }^{19}$ have suggested that in patients who undergo posterior arachnoidal dissection, failure to achieve reduction of the syrinx was due to inadequate dissection of the intradural adhesions. We believe that this may be a contributing factor; however, in some patients with a fixed spinal deformity, causing extradural compression, not removing the displaced ventral bone may be critical to poor surgery-related outcome.

\section{Analysis of Surgical Options}

Although some surgeons still favor performing procedures in which the syrinx is drained, many neurosurgeons now choose subarachnoid space reconstructive procedures as the first line of surgical management procedures in the treatment of PTS., ${ }^{2,10,13,18,21}$ The concept driving this change is the importance now placed on correcting the anatomical abnormalities that contribute to the filling mechanism of the syrinx, which theoretically treats the source of the problem and potentially yields improved long-term results. Although the majority of patients with PTS primarily have intradural obstruction, which responds well to expansile duraplasty, we purport that a significant number of patients have a spinal deformity with extradural osseous compression that is the primary pathological entity or that is at least an important cofactor. Spinal deformity in which significant stenosis is present can contribute to the formation of PTS several mechanisms by: 1) direct 
impingement and obstruction of the subarachnoid space, 2) tethering of the spinal cord at the fracture site, and 3) spinal cord ischemia due to direct compression. Therefore, in some cases of PTS correction of the spinal deformity is required to abolish the mechanism by which the syrinx becomes filled and to reconstruct the subarachnoid space adequately.

Our mean follow-up period of 38 months is relatively short with regard to determining the natural history of PTS, a condition in which late-onset deterioration may occur whether or not surgical intervention is performed. However, based on our early experience we believe that ventral extradural decompressive surgery should be the initial procedure for the treatment of all patients with PTS in whom a significant ventral bone lesion is demonstrated. This procedure is associated with very little risk of CSF leakage and, being extradural, the risk of increased neurological deficit or stimulating additional intradural adhesion formation is very low when compared with that associated with posterior subarachnoid space reconstruction. Furthermore, the extradural approach may be technically simpler than an intradural one, even if a transthoracic approach is necessary.

\section{CONCLUSIONS}

Posttraumatic syringomyelia, although uncommon, is a cause of delayed-onset neurological deterioration in spinal cord-injured patients, and it is becoming more frequently recognized due to physician awareness and the availability of MR imaging. It is considered to be caused by the obstruction of subarachnoid CSF flow, and recent surgical treatment has evolved toward procedures that reestablish subarachnoid CSF flow. The anatomical abnormalities that contribute to the pathogenesis of PTS can be intradural and/or extradural in origin. In cases of significant spinal canal stenosis or an osseous lesion the patients should be considered initially for decompression of the extradural mass, which may result in reduction of the syrinx and resolution of neurological deficits. Our experience justifies making an effort to achieve anatomical correction of acute- and late-onset traumatic spinal deformities for the management of this delayed complication.

\section{Acknowledgments}

The authors wish to thank Marsha Langaard and Gena Behnke for their assistance in preparing this manuscript. The medical illustrations were created by Josh Emerson.

\section{References}

1. Abel R, Gerner HJ, Smit C, et al: Residual deformity of the spinal canal in patients with traumatic paraplegia and secondary changes of the spinal cord. Spinal Cord 37:14-19, 1999

2. Batzdorf U, Klekamp J, Johnson JP: A critical appraisal of syrinx cavity drainage procedures. J Neurosurg 89:382-388, 1998
3. Biyani A, el Masry WS: Post-traumatic syringomyelia: a review of the literature. Paraplegia 32:723-731, 1994

4. Edgar R, Quail P: Progressive post-traumatic cystic and noncystic myelopathy. Br J Neurosurg 8:7-22, 1994

5. el Masry WS, Biyani A: Incidence, management, and outcome of post-traumatic syringomyelia. In memory of Mr. Bernard Williams. J Neurol Neurosurg Psychiatry 60:141-146, 1996

6. Falci S, Holtz A, Akesson E, et al: Obliteration of a posttraumatic spinal cord cyst with solid human embryonic spinal cord grafts: first clinical attempt. J Neurotrauma 14:875-884, 1997

7. Hida K, Iwasaki Y, Imamura H, et al: Posttraumatic syringomyelia: its characteristic magnetic resonance image findings and surgical management. Neurosurgery 35:886-891, 1994

8. Hormigo A, Lobo-Antunes J, Bravo-Marques J, et al: Syringomyelia secondary to compression of the cervical spinal cord by an extramedullary lymphoma. Neurosurgery 27:834-836, 1990

9. Klekamp J, Batzdorf U, Samii M, et al: The surgical treatment of Chiari I malformation. Acta Neurochir 138:788-801, 1996

10. Klekamp J, Batzdorf U, Samii M, et al: Treatment of syringomyelia associated with arachnoid scarring caused by arachnoiditis or trauma. J Neurosurg 86:223-240, 1997

11. Kramer KM, Levine AM: Posttraumatic syringomyelia: review of 21 cases. Clin Orthop 334:190-199, 1997

12. La Haye PA, Batzdorf U: Posttraumatic syringomyelia. West J Med 148:657-663, 1988

13. Levi AD, Sonntag VK: Management of posttraumatic syringomyelia using an expansile duraplasty. A case report. Spine 23: 128-132, 1998

14. Menezes AH: Primary craniovertebral anomalies and the hindbrain herniation syndrome (Chiari I): data base analysis. Pediatr Neurosurg 23:260-269, 1995

15. Milhorat TH, Johnson WD, Miller JI, et al: Surgical treatment of syringomyelia based on magnetic resonance imaging criteria. Neurosurgery 31:231-245, 1992

16. Perrouin-Verbe B, Lenne-Aurier K, Robert R, et al: Post-traumatic syringomyelia and post-traumatic spinal canal stenosis: a direct relationship: review of 75 patients with a spinal cord injury. Spinal Cord 36: 137-143, 1998

17. Schurch B, Wichmann W, Rossier AB: Post-traumatic syringomyelia (cystic myelopathy): a prospective study of 449 patients with spinal cord injury. J Neurol Neurosurg Psychiatry 60: 61-67, 1996

18. Sgouros $\mathrm{S}$, Williams B: A critical appraisal of drainage in syringomyelia. J Neurosurg 82:1-10, 1995

19. Sgouros S, Williams B: Management and outcome of posttraumatic syringomyelia. J Neurosurg 85:197-205, 1996

20. Squier MV, Lehr RP: Posttraumatic syringomyelia. J Neurol Neurosurg Psychiatry 57:1095-1098, 1994

21. Williams B: Pathogenesis of post-traumatic syringomyelia. $\mathbf{B r}$ J Neurosurg 6:517-520, 1992

22. Williams B: Post-traumatic syringomyelia, an update. Paraplegia 28:296-313, 1990

23. Williams B, Terry AF, Jones F: Syringomyelia as a sequel to traumatic paraplegia. Paraplegia 19:67-80, 1981

Manuscript received December 6, 1999.

Accepted in final form February 1, 2000.

Address reprint requests to: J. Patrick Johnson, M.D., UCLA Division of Neurosurgery, Box 956901, Los Angeles, California 90095-6901. 